\title{
Relatives and friends group in a psychiatric ward
}

\author{
D GOLDMEIER, D HOLLANDER, M J SHEEHAN
}

British Medical fournal, 1979, 1, 932-934

\section{Summary and conclusions}

To enable relatives and friends of psychiatric patients in a 30-bed acute admission ward to meet the staff team together a relatives and friends group was initiated. The group was intended to provide a forum for questions about the patients' illness and treatment; allow feelings, such as anger and anxiety, about the patient's illness to be expressed; and enable relatives and friends to share their experiences and offer advice and support to each other. A study was designed to assess the feasibility of the group scheme. Relatives and friends were invited to the day room of the admission ward each Wednesday evening for one hour. The visitors chose their own topics for discussion and the staff team tried to answer questions and to facilitate a free exchange of views. An account of each meeting was documented, including details of attenders, the nature of the topics raised, and a simple measurement of some of the emotions expressed by the visitors.

Overall, the effect of the meetings seemed positive and productive, for they allowed relatives and friends to ask questions about many topics, to express pent-up emotions, and to gain a better understanding and tolerance of the patient's illness. The relatives and friends group has become an integral part of treatment on the wards. Now that the feasibility of such a group is established, further studies are required to evaluate its efficacy.

Friern Hospital, London N11 3BP

D GOLDMEIER, MD, MRCP, honorary senior registrar

D HOLLANDER, MD, MRCPSYCH, consultant psychiatrist

M J SHEEHAN, BA, MSC, clinical psychologist

\section{Introduction}

Mental illness puts pressure on relatives and friends of the patient, particularly if the condition is severe and requires admission to hospital. Regular liaison between psychiatrists, other professional workers, and the family may be valuable to all concerned but the health workers are rarely all available at the same time to meet the family and friends of the patient. We thought that a multidisciplinary professional team forming a group with patients' relatives and friends would allow the relatives and friends to meet the staff team together; provide a forum for questions about the patient's illness and treatment; allow relatives and friends to express feelings, such as anger and anxiety, about the patient's illness; and allow them to share their experiences and offer advice and support to each other. We set out, therefore, to test the feasibility of running such a group.

\section{Methods}

All relatives and friends who came to our 30-bed acute admission ward were given details of the time and venue (7 00-8 $00 \mathrm{pm}$ every Wednesday in the day room of the admission ward) of the relatives and friends group. The purpose of the group was explained before the initial attendance-namely, to help relatives and staff to understand more about the patient's illness and to discuss problems about the illness and its treatment. The number of participants and frequency of attendance (even after the patient was discharged) were not limited. The professional team within the group consisted of a consultant, a registrar, two or three nurses, a social worker, a psychologist, an occupational therapist, and occasionally medical students.

Two months before the relatives and friends group was begun the idea was raised and discussed weekly with all patients on the ward, and all supported the idea. As patients were admitted, they were told about the group and encouraged to invite their relatives and friends. None raised any objections. The patients could neither see nor hear the group in progress but knew that it was taking place and were told that its purpose was to help them through discussion between relatives and staff.

Relatives and friends were generally allowed to choose the subjects for discussion and were encouraged to seek advice from other relatives 
and staff. Nevertheless, if strong feelings threatened to become overwhelming or if an important topic had arisen in the wards the staff took a more directive role. Staff explained practical issues, were generally supportive, and made no psychodynamic interpretations. After each session the staff met and discussed the group. Details of the first 30 consecutive groups were recorded from 11 May 1977. The information recorded included details of the relatives and friends who attended and the nature of the topics raised.

We also tried to assess simply the considerable degree of emotion expressed during the group discussions by combining hostility, fear, and anxiety as the first category and expressed guilt as the second. Relatives and friends who spoke in overtly hostile terms (directed at staff, patients, or the group members) or who appeared fearful or anxious about issues concerning the patient were given a score of one unit of hostility, fear, and anxiety (HFA) for that session. Expressed guilt was likewise noted and scored.

\section{Results}

\section{DIAGNOSTIC CATEGORIES}

The thirty-two patients whose relatives and friends came to the group fell into the following five diagnostic categories, some having more than one diagnosis (numbers of patients in parentheses): nonaffective psychosis (8); affective disorder (20); personality disorder (12); organic illness (1); alcoholism (2).

\section{ATTENDANCE}

Fifty relatives who were associated with the 32 patients attended the group over 30 weeks. The mean attendance at each session for the relatives was $6 \cdot 3 \pm \mathrm{SD} 2 \cdot 46$ (range 2-12). Twenty-one relatives attended once; 10 attended twice; five attended three times; five attended four times; and nine attended more than five times. The relatives were divided into two groups, according to attendances. Group A comprised the 41 relatives who attended up to four meetings, and group $B$ comprised the nine who attended five or more.

\section{TOPICS DISCUSSED AT THE MEETING}

The relatives and friends entered into the discussions surprisingly easily, and many issues were raised during the 30 weeks. Some of the recurrent subjects included: explanation of various mental illnesses; electric convulsion treatment; drug treatment and side effects; electroencephalograms; patients' financial problems; holidayswhether to take the patient or not; coping with patients' children; explanation of why some patients are nursed in pyjamas and dressinggown; whether to tell patients of problems facing family and friends outside hospital, such as losing a job or deaths of other relatives; what relatives should say if patients asked about their illness; the patients' activities during the day; whether to trust patients to take medication as outpatients; coping with medical leave; the desire of friends and relatives to devote all their lives to the patient; coping with aggression at home; discussion of discharge dates; allowing patients home when relatives think that they should be kept in hospital; unexpected visits home by patients.

\section{ASSESSMENT OF EXPRESSED EMOTION}

The table shows the total number of units of HFA in groups $A$ and $B$ over 30 sessions. On the two occasions that a patient came into the meeting the HFA score reached a high level. Five units of guilt were recorded (weeks 19,21,22, 24, and 28), and four of the five were expressed by group B members (those who attended five times or more).

\section{Other observations}

Other observations made by the staff of the dynamics of the group are described below.

Relatives-The directness of the relatives' questions to one another and the resulting discussions provided a shortcut to highlighting certain sensitive areas-for example, parental possessiveness, rejection and avoidance by friends and relatives, and recognition of illness and stress in each other. Apparent lack of inhibition in people who had not met before led to early and fruitful discussion of pressing problems. Often relatives were more ready to accept the realities of the illness and reassurance from other relatives than from the staff. The relatives encouraged each other to have lower expectations of the patients. Those relatives and friends who had attended five sessions or more were particularly helpful in forming a cohesive force and encouraging discussion.

Staff-As well as identifying illness in the relatives, the staff were more easily able to understand the problems faced by relatives and patients before, during, and after the patient's admission to hospital.

Patients-The relatives and friends meeting was made a subject for debate in the patients' morning group. They seemed generally to accept and welcome it. During the relatives' meeting the patients stayed outside and only twice in 30 weeks did a patient come into the meeting.

\section{Discussion}

Although attendance was voluntary, the group was consistently attended by relatives and friends. Those who attended five times or more constituted a minority of the total attending over 30 weeks, but were a major force in any particular group. Their effect on relatives who were newer to the group was positive and beneficial. Our figures suggest that the patients of relatives in group B were in hospital for longer than those of relatives in group A. Group A constituted $82 \%$ of the total number of relatives attending the meetings. Even though they attended fewer than five meetings, we believe that they derived benefit from them, perhaps by brief catharsis, concentrated fact-finding, and reassurance from other relatives. Nevertheless, they may not have gained much benefit and thus attended a few times only.

\section{AIMS AND OBJECTIVES}

How the specific aims and objectives of the meetings were met is discussed below.

Enabling relatives and friends to meet the staff team togetherProblems about patients were discussed by the group in depth. For example, when the patients' activities during the day were being discussed the occupational therapist was able to give expert advice, supported by nurses and other staff.

Providing a forum for questions about the patient's illness and treatment-A wide range of topics was covered at the meetings and many questions came up time and time again. This suggests that such a forum was indeed warranted. Relatives and friends seemed to come to meetings with one pressing problem needing discussion. Problems raised by any one relative or friend, however, were generally conceded to be common to all the relatives.

Allowing expression of feelings about the patient's illness-Over the 30 weeks HFA scores were relatively constant and similar in the two groups (table). Such feelings expressed by one group ing up to four meetings, group $B(n=9)$ those attending five or more

\begin{tabular}{lccccccccccccccccccccccccccccccccc}
\hline & Week: & 1 & 2 & 3 & 4 & 5 & 6 & 7 & 8 & 9 & 10 & 11 & 12 & 13 & 14 & 15 & 16 & 17 & 18 & 19 & 20 & 21 & 22 & 23 & 24 & 25 & 26 & 27 & 28 & 29 & 30 \\
\hline Group A & & 1 & 2 & 2 & 1 & 2 & 1 & 2 & 3 & 1 & & 3 & & 1 & 2 & 2 & 1 & 2 & 2 & 2 & 2 & 2 & 2 & 2 & 2 & 2 & 1 & 1 & 2 & 1 & $3^{*}$ \\
Group B & & & 2 & & 1 & 2 & $5^{*}$ & 2 & & 2 & 2 & & 2 & 1 & 2 & 2 & 1 & & 2 & 2 & 2 & 1 & & & 2 & 1 & 1 & 1 & & 1 & 1
\end{tabular}


to the exclusion of the other seemed to enable the less frequent attenders to express pent-up emotions. Possibly, when the needs of group A members were less pressing the more frequent attenders felt that they could discharge negative, pent-up feelings. If the relatives' feelings of fear, hostility, and anger are channelled away from the patient into the group the intensity of these possibly destructive emotions may be lessened. Klein ${ }^{1}$ discussed the close connection between anxiety and guilt, and postulated that expressed anger may result in persecutory feelings. Hostility in the relatives and friends is understandable, for their livelihood is threatened, their position in the community jeopardised, and much of their time spent patching up the damage caused by the actions of their mentally ill relatives. They may fantasise that the patients will develop an angry response to the hostility-a persecutory anger that will be directed back at the person who expressed the hostility. This, together with the patient's illness-provoked actions (which may be frightening), makes the anxiety all the more comprehensible. Such feelings may be displaced on to other relatives and friends or staff in the group discussions.

Guilt-When the relatives and friends consider that the patient's condition has improved, either through hospital treatment or lessening of symptoms, their hostility, fear, and anxiety will decrease. They may then feel that the patient's illness and need for hospital treatment were partly due to their hostile feelings and actions, and therefore feel and express guilt. Few of the relatives and friends expressed such guiit; but, interestingly, four of the five who did were in group B. They thus had time and opportunity to express hostility, fear, and anxiety; see the patient improve; and develop guilt.

Enabling relatives and friends to share their experiences and offer advice and support to each other-Despite initial shyness and reluctance to talk at the start of the meetings, by the end of each session more ground had been covered than would have been possible in a one-to-one meeting, and both relatives and staff learnt more about the patient, his illness, and his problems in society and those of people close to him. Accepting poor prognosis, uncertainty about the course of the illness, and consolation at times of frightening or upsetting events seemed to be matters that other relatives and friends could help and sympathise with more easily and efficiently than could the staff. The staff were then free to take on a more directive role in the group.

Cartwright and Zander ${ }^{2}$ believe that a group of people who are dependent on each other develop a working capacity that is greater than that of the individual group members. Our group could therefore be seen as a time-saving device, in that less time was spent talking to relatives individually. More importantly, the relatives perhaps gained a greater understanding and tolerance of the patient's illness and capacities in the group discussions than they would have gained by individual counselling. Our study was not designed to measure the benefit of the relatives and friends group to individual patients, but responses from both patients and their relatives have been favourable and have encouraged us to continue. Further work is needed to evaluate the scheme objectively.

We thank Dr J T Bruce for agreeing to the starting of the group.

\section{References}

1 Klein, M, International fournal of Psycho-analysis, 1948, 3, 29.

2 Cartwright, D, and Zander, A, Group Dynamics. London, Harper and Row, 1953.

(Accepted 29 Fanuary 1979)
How should a schizophreniform psychosis occurring in a patient with epilepsy be treated?

Two forms of schizophreniform psychosis in patients with epilepsy are recognised: firstly, those that occur in a state of clouded consciousness, are closely related to the seizure, and are associated with an abnormal EEG, dominated by delta activity; secondly, those that occur in a state of clear consciousness between seizures and where the EEG is likely to be normal or to show the usual epileptic features. These latter are commonly associated with temporal lobe epilepsy. The treatment of the psychosis is symptomatic as with any other psychotic illness. Admission to hospital is usually required, and a course of phenothiazines is given until the acute features of the illness remit. If the psychosis is associated with a seizure then the episode should clear up within a few days or at the most a week or so. The interictal psychosis of temporal lobe epilepsy may become chronic and need long-term treatment with phenothiazines, and some of these patients show organic deterioration. In both cases anticonvulsant treatment is continued so as to control the epileptic attacks.

Are there any health risks from the Scandinavian-style wood-burning stoves that are becoming popular?

"Many woodburners point out that a $\log$ fire is virtually nonpolluting," claimed an article on "The Back-to-Wood Boom" in Time. ${ }^{1}$ The combustion of materials containing carbon, however, inevitably leads to the formation of many compounds of environmental and toxicological significance, which undergo physical and chemical changes on emission into ambient air. ${ }^{2}$ Emissions comprise both particulate matter and gaseous components, of which polycyclic aromatic hydrocarbons, notably benzopyrene, have attracted particular interest as mutagenic and carcinogenic compounds. The amount of individual components and the composition of the emission vary depending on the fuel, firing conditions, and the flue factors. With the advent of short-term testing for detection of potential pollutants, it has become possible to screen complex samples routinely for the presence of genotoxic compounds. ${ }^{2}$ The most authoritative recent review of such work, however, concluded, "it is presently neither possible to say that we are aware of all responsible agents or classes of compounds, nor possible to say that any of the known classes of compounds are major contributors to the total hazard ... much is unknown about genotoxic compounds... [further] studies are needed." 3 Nevertheless, a wood-burning stove probably constitutes less potential hazard than stoves burning other solid fuels, and the best general advice is to ensure optimum functioning of the stove with the maintenance of a constant draught and efficient discharge and dispersal of emissions.

1 Time Magazine, 5 December 1977, $\mathrm{p} 103$

: Löfroth, G, Chemosphere, 1978, 7, 791 .

Ehrenberg, L, and Löfroth, G, In the Beijer Institute International Seminar on Impacts and Risks of Energy Strategies: Their Analysis and Role in Manage-
ment. Stockholm, 1978 .

What is the likely psychological effect on a surviving twin accused by her mother as having been partly responsible for the other twin's (brother) death?

The reason for a mother accusing a surviving twin is in itself of interest. Unresolved mourning for the dead child may give rise to resentment at the survivor for being alive. A dead child tends to be idealised. The seemingly less desirable survivor suffers by comparison and is blamed. Even without a mother's blame a surviving twin tends unconsciously to feel some responsibility for the death of its twin, partly because of preceding jealousy, but also because of irrational resentment with the dead twin for dying, and so causing much family unhappiness. The child will feel guilty and depressed, and may show excessive concern for the health of live children. It is hard to be the surviving twin of a stillbirth for there is a sense of having survived at the expense of the other, which can give rise to guilt, depression, and overconcern for the underdog. The sense of guilt may make it difficult for the surviving twin to be successful and happy at school and in adult life. The desire to make amends in later life may lead to the choice of a spouse who is seen as a weakling needing to be cared for. 